\title{
NEW BOOKS
}

Joseph Llewellyn Whrte. The Flight Pattern to Eternity. Philosophical Library Inc., N. York. 1968. Pp. 120. \$4.00.

T. C. Williams. The Concept of the Categorical Imperative. Clarendon Press: Oxford University Press. 1968. Pp. 142. 32s.

Robert Wolfr (Ed.). Kant. Macmillan. 1968. First published U.S.A. 1967. Pp. xxiii + 416. 36s. Papermac 12s. $6 \mathrm{~d}$.

Richard Wollherm. Art and its Objects. Harper \& Row Ltd. 1968. Pp. 152. 18s.

William Young. Foundations of Theory. Craig Press. 1967. Pp. xi + 121. \$3.75.

Guglialmo Forni. Il Sogno Finito. Società editrice Il Mulino, Bologna. 1967. Pp. 276. L. 3000.

Einar Gjerstad and others. Les Origines de la République Romaine. Heffer \& Sons. 1968. Pp. 389. 78s.

Hennz Hector. Vom Sien. (No publisher's name.) Stockholm. 1967. First published 1963. Pp. 80. Kr. 10.

Jacques Herbrand. Ecrits Logiques. Presses Universitaires de France. 1968. Pp. 243. 24F.

Jean Lacroix (Ed.). Les Hommes devant L'Echec. Presses Universitaires de France. 1968. Pp. 240. 16F.

Jean Piaget. Le Structuralisme. Presses Universitaires de France. 1968. Pp. 124. No price given.

R. Polin. Ethique et Politique. Editions Sirey. Paris. 1968. Pp. 257. 25F.

Joseph Rassam. La Métaphysique de Saint Thomas. Presses Universitaires de France. 1968. Pp. 144. 6F.

J. L. Rigal. Le Temps et La Pensée Physique Contemporaine. Dunod, Paris. 1968. Pp. 184. 16.50F.

\section{CORRIGENDUM}

Line 24 on page 218 in the July number of this volume of Prinosopry should read as follows:

naturally out of matter itself lifeless or that consciousness and intelli- 